\title{
Cannabis nur aus der Apotheke?
}

KOMMENTAR

Eine Therapie mit Cannabis bzw. CannabisExtrakten oder -Einzelstoffen ist in Deutschland nur begrenzt erlaubt. Seit Mai 2011 dürfen zugelassene Fertigarzneimittel auf Basis von Cannabis hergestellt und von Ärzten auf Betäubungsmittel-Rezept verschrieben werden. Zugelassen ist bisher lediglich ein Gesamtextrakt aus Blüten und Blättern von Cannabis sativa L. unter dem Namen „Sativex ${ }^{\circledR “}$ für Patienten, die an schweren spastischen Lähmungen und Krämpfen aufgrund einer Multiplen Sklerose leiden. Bereits seit Längerem kann Patienten der insbesondere in den USA und Kanada zugelassene Wirkstoff Tetrahydro-

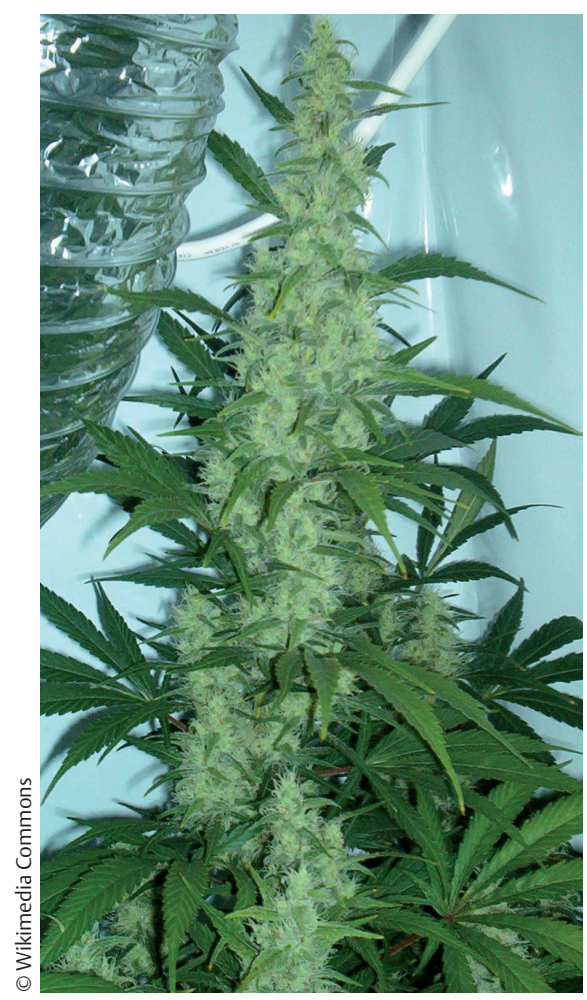

Selbstversorgung von Schwerkranken kann nicht die Lösung sein. cannabinol als Monosubstanz mit den INNBezeichnungen Dronabinol bzw. Nabilon verschrieben werden. Beide sind auf Basis des Betäubungsmittelgesetzes verkehrsund verschreibungsfähig. Für die relativ hohen Kosten (ca. 700.- €/Monat) müssen die Patienten in der Regel aber selbst aufkommen. Alternativ können Patienten bei der Bundesopiumstelle eine Ausnahmeerlaubnis zum Erwerb von Cannabis-Blüten oder -extrakten ,im Rahmen einer medizinisch betreuten und begleiteten Selbsttherapie“ beantragen.

Das sog. „Cannabis-Urteil“ des Kölner Verwaltungsgerichts hat vor wenigen Wochen für Wirbel gesorgt. Das Gericht hatte über die Klage von 5 chronisch kranken Schmerzpatienten zu befinden. Die Kläger hatten zwar alle die Erlaubnis vom BfArM, Cannabis-Blüten aus der Apotheke zu erwerben und zu konsumieren. Den Eigenanbau in der Wohnung hatte das Bundesinstitut allerdings untersagt. Die gegen die Ablehnung gerichteten Klagen hatten nun vor dem Verwaltungsgericht in 3 Fällen überwiegend Erfolg. Die Kölner Richter entschieden, dass der Cannabis-Eigenanbau im Grundsatz verboten bleibt, er könne aber unter mehreren Bedingungen „in besonders gelagerten Ausnahmefällen“ zum therapeutisch erforderlichen Eigenkonsum erlaubt werden. Wenn Kranken sonst nichts gegen ihre Schmerzen helfe und Cannabis aus der Apotheke für sie unerschwinglich sei, dürften sie es als Notlösung selbst anbauen.

In 3 Fällen hielt das Gericht die Voraussetzungen für einen zulässigen Eigenanbau grundsätzlich für gegeben. $\mathrm{Zu}$ diesen gehört, dass der Patient austherapiert ist, es keine Behandlungsalternativen gibt und der Preis in der Apotheke für ihn unerschwinglich ist. Die genauen Modalitäten des Cannabis-Anbaus könnten nach Meinung der Richter durch Auflagen bestimmt werden. Zwei Klagen wies das Gericht allerdings ab. In dem einen Fall hielten die Richter einen gegen den Zugriff Unbefugter gesicherten Anbau aufgrund der Wohnsituation des Klägers nicht für möglich. Im anderen Fall ging die Kammer davon aus, dass der Patient noch nicht alle zumutbaren Alternativen für die Behandlung seiner Krankheit ausgeschöpft hat.

Die Auseinandersetzung um den privaten Eigenanbau von Cannabis zu Therapiezwecken spitzt sich zu. Es ist nicht das erste Mal, dass ein Gericht sich mit einer derartigen Klage befassen musste. Am 11. Juni 2014 entschied das Oberverwaltungsgericht in Münster (OVG) in einem Berufungsverfahren, dass der Anbau von Cannabis zur Selbsttherapie im Einzelfall zulässig sei. Ein bedeutender Unterschied zum Kölner Urteil ist jedoch, dass das OVG die letzte Entscheidung über eine Genehmigung dennoch weiterhin beim BfArM sah. Von den Kölner Richtern wurde das BfArM beauftragt, unter Beachtung der Auffassung des Gerichtes, die Anträge der Kläger erneut zu bescheiden. Das BfArM nutzte nun die Rechtsmittel und hat Berufung eingelegt.

Cannabis ist die am häufigsten konsumierte illegale Droge in Deutschland. Lediglich rund 270 Menschen bundesweit hat das BfArM ausnahmsweise den Cannabis-Konsum nach Kauf aus der Apotheke gestattet. Eine Eigenproduktion hält die Behörde für gesundheitlich riskant, unerwünschte $\mathrm{Ne}$ benwirkungen seien möglich. Bisher hatte es noch keine Erlaubnis für einen privaten Anbau durch das BfArM gegeben.

Es bleibt abzuwarten und zu hoffen, dass schnell eine Lösung im Interesse einer medizinisch sinnvollen und qualitätsgesicherten Versorgung der Patienten erreicht wird. Die Plantage daheim für jeden Schwerkranken kann nicht die Lösung sein.

Prof. em. Dr. Karen Nieber

Bahnhofstr. 8

39245 Gommern

nieber@rz.uni-leipzig.de 